\title{
A Method of Evaluating Certain Determinants
}

\author{
By J. L. Burchnall.
}

(Received 22nd January, 1952. Read 7th March, 1952.)

1. Let $\left[a_{s t}\right](s, t=0,1, \ldots, n)$ be a square matrix of order $n+1$ and determinant $\left|a_{s t}\right|$ and suppose that by repeated "isolation" of the variables the corresponding bilinear form has been expressed as

$$
\sum_{s=0}^{n} \sum_{t=0}^{n} a_{s t} X_{s} Y_{t}=\sum_{r=0}^{n} c_{r}\left\{\sum_{s=r}^{n} p_{r s} X_{s}\right\}\left\{\sum_{t=r}^{n} q_{r t} Y_{t}\right\}
$$

where, for all $r$,

$$
p_{r r}=q_{r r}=1
$$

Then

$$
\left|a_{s t}\right|=\prod_{r=0}^{n} c_{r}
$$

Now (1) implies, and is implied by, the identities

$$
a_{s t}=\sum_{r=0}^{\min (s, t)} c_{r} p_{r s} q_{r t} \quad(s, t=0,1, \ldots, n) .
$$

Thus, from any known identity of the form (4), subject to the condition (2), we may at once infer, using (3), the value of the corresponding determinant $\left|a_{r s}\right|$.

I have elsewhere ${ }^{1}$ applied this method, without explicit formulation, to evaluate the determinants in which

(i) $a_{s t}=F(a,-s-t ; c ; x)$,

(ii) $a_{s t}=\left(\begin{array}{c}s+t+2 \lambda-1 \\ s+t\end{array}\right)^{-1} P_{s+t}^{\lambda}(x)$,

where $F$ is the hypergeometric function and $P_{n}{ }^{\lambda}$ the ultra-spherical polynomial. I collect here some further instances, in part I believe new, in part furnishing alternative proofs of results given previously by myself and others ${ }^{2}$.

1 (1) $\$ 6$.

2 (2) and (3) for the formulae of $\S \S 2,3$, 
2. Let

$$
H_{n}(x)=\exp \left(\frac{1}{2} x^{2}\right)(-d / d x)^{n} \exp \left(-\frac{1}{2} x^{2}\right) .
$$

be the Hermitian polynomial of degree $n$. Then ${ }^{1}$

$$
H_{s+l}(x)=\sum_{r=0}^{\min (s, t)}(-1)^{r} r !\left(\begin{array}{l}
s \\
r
\end{array}\right)\left(\begin{array}{l}
t \\
r
\end{array}\right) H_{s-r}(x) H_{t-r}(x)
$$

which we identity with (4) on letting

$$
c_{r}=(-1)^{r} r !, p_{r s}=\left(\begin{array}{l}
s \\
r
\end{array}\right) H_{s-r}(x), q_{r t}=\left(\begin{array}{l}
t \\
r
\end{array}\right) H_{t-r}(x) .
$$

Since (2) is clearly satisfied we have at once

$$
\left|H_{s+l}(x)\right|=(-1)^{i n(n+1)} \prod_{r=1}^{n}(r !) .
$$

3. Let $P_{n}(x)$ be Legendre's polynomial, let $2 u=x+1,2 v=x-1$ and $k_{p}{ }^{q}$ be the coefficient of $z^{2 p-q}$ in the expansion of

$$
\{(z+u)(z+v)\}^{p} .
$$

Evidently $k_{p}{ }^{0}=1$ for all $p$.

Now ${ }^{2}$

$$
P_{s+l}(x)=k_{s}^{s} k_{t}^{t}+2 \sum_{r=1}^{\min (s, t)}(u v)^{r} k_{s}^{s-r} k_{t}^{s-r}=\sum_{r=0}^{\min (s, t)} c_{r} p_{r s} q_{r t}
$$

with

$$
c_{0}=1, c_{r}=2(u v)^{r} \quad(r>1) .
$$

Hence

$$
\left|P_{s+\ell}(x)\right|=2^{n}(u v)^{\sharp n(n+1)}=2^{-n^{2}}\left(x^{2}-1\right)^{\ddagger n(n+1)} .
$$

4. Let

$$
\begin{aligned}
& F^{(1)}(s, t)=\sum_{m=0}^{s} \sum_{n \cdots 0}^{t} \frac{(a)_{m+n}(-s)_{m}(-t)_{n}}{m ! n !(c)_{m+n}} x^{m} y^{n} \\
& F^{(2)}(s, t)=\sum_{m=0}^{s} \sum_{n=0}^{t} \frac{(a)_{m+n}(-s)_{m}(-t)_{n}}{m ! n !(c)_{m}\left(c^{\prime}\right)_{n}} x^{m} y^{n} \\
& F^{(3)}(s, t)=\sum_{m=0}^{s} \sum_{n-0}^{t} \frac{(a)_{m}\left(a^{\prime}\right)_{n}(-s)_{m}(-t)_{n}}{m ! n !} x^{m} y^{n}
\end{aligned}
$$

where

$$
(k)_{r}=k(k+1) \ldots(k+r-1) \text {. }
$$

${ }^{1}(4), 10(5)$, with a change of notation.

2(2), 230 (17). 
These are, of course, instances of Appell's hypergeometric functions of two variables in which a pair of numerator parameters have been replaced by negative integers and, in consequence, the functions reduce to polynomials in two variables of total degree $s+t$.

If now we adapt to these special parameters known expansions ${ }^{1}$ of Appell's functions we have

$$
\begin{aligned}
& F^{(1)}(s, t)=\sum_{r=0}^{\min (s, t)} \frac{r !(a)_{r}(c-a)_{r}}{(c+r-1)_{r}(c)_{2 r}}(x y)^{r}\left(\begin{array}{l}
s \\
r
\end{array}\right)\left(\begin{array}{l}
t \\
r
\end{array}\right) F\left[\begin{array}{c}
a+r,-s+r, \\
c+2 r,
\end{array}\right] \\
& \times F\left[\begin{array}{c}
a+r,-t+r, \\
c+2 r,
\end{array}\right] \\
& F^{(2)}(s, t)=\sum_{r=0}^{\min (o, t)} \frac{r !(a)_{r}}{(c)_{r}\left(c^{\prime}\right)_{r}}(x y)^{r}\left(\begin{array}{l}
s \\
r
\end{array}\right)\left(\begin{array}{l}
t \\
r
\end{array}\right) F\left[\begin{array}{cc}
a+r,-s+r, & x \\
c+r, &
\end{array}\right] \\
& \times F\left[\begin{array}{c}
a+r,-t+r, \\
c^{\prime}+r,
\end{array}\right]
\end{aligned}
$$

and

$$
\begin{aligned}
F^{(3)}(s, t)=\sum_{r=0}^{\min (s, t)} \frac{(-1)^{r}(a)_{r}\left(a^{\prime}\right)_{r}}{(c+r-1)_{r}(c)_{2 r}}(x y)^{r}\left(\begin{array}{l}
s \\
r
\end{array}\right)\left(\begin{array}{l}
t \\
r
\end{array}\right) & F\left[\begin{array}{cc}
a+r,-s+r, & x \\
c+2 r,
\end{array}\right] \\
& \times F\left[\begin{array}{cc}
a^{\prime}+r,-t+r, & y \\
c+2 r, & y
\end{array}\right],
\end{aligned}
$$

where $F$ is in every case the ordinary hypergeometric function.

Each of these formulae is of the form (4); the condition (2) is satisfied and we deduce

$$
\begin{aligned}
& \left|F^{(\mathbf{1})}(s, t)\right|=(x y)^{i n(n+1)} \prod_{r=1}^{n}\left[\frac{r !(a)_{r}(c-a)_{r}}{(c+r-1)_{r}(c)_{2 r}}\right] \\
& \left|F^{(2)}(s, t)\right|=(x y)^{\ddagger(n(n+1)} \prod_{r=1}^{n}\left[\frac{r !(a)_{r}}{(c)_{r}\left(c^{\prime}\right)_{r}}\right]
\end{aligned}
$$

and

$$
\mid F^{(3)}(s, t)=(-x y)^{i n(n+1)} \prod_{r=1}^{n}\left[\frac{r !(a)_{r}\left(a^{\prime}\right)_{r}}{(c+r-1)_{r}(c)_{2 r}}\right] .
$$

${ }^{1}(5)$, pp. 253-4 (30), (26), (28). 
If we recall that, when $y=x, F^{(1)}$ reduces to an ordinary hypergeometric function ${ }^{1}$ we see that (9) includes as a special case

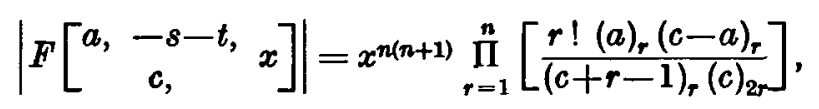

which I have given elsewhere 2 .

Formulae of more apparent complexity may be obtained by increasing the number of the non-integral parameters involved and employing for example the formulae (11)-(13) of (6). Alternatively we may consider degenerate hypergeometric functions and from ${ }^{3}$

$$
\begin{aligned}
& { }_{1} F_{1}[-8-t ; c ; x] \\
& =\sum_{r=0}^{\min (e, t)} \frac{(-1)^{r} r !}{(c+r-1)_{r}(c)_{2 r}}\left(\begin{array}{l}
8 \\
r
\end{array}\right)\left(\begin{array}{l}
t \\
r
\end{array}\right){ }_{1} F_{1}[-s+r ; c+2 r ; x]_{1} F_{1}[-t+r ; c+2 r ; x]
\end{aligned}
$$

obtain the result

$$
\left|{ }_{1} F_{1}[-s-t ; c ; x]\right|=\left(-x^{2}\right)^{\dagger(n(n+1)} \prod_{r=1}^{n}\left[\frac{r !}{(c+r-1)_{r}(c)_{2 r}}\right] .
$$

5. Inspection of the formulae (9)-(11) shows that the right-hand side of each arises from the terms of highest degree only in each element of the determinant on the left. If we take these terms only and recall that

we find that

$$
\left|(-1)^{s+r} a_{s i}\right|=\left|a_{s t}\right|
$$

$$
\begin{gathered}
\left|\frac{(a)_{c+l}}{(c)_{s+l}}\right|=\prod_{r=1}^{n}\left[\frac{r !(a)_{r}(c-a)_{r}}{(c+r-1)_{r}(c)_{2 r}}\right], \\
\left|\frac{(a)_{s+l}}{(c)_{s}\left(c^{\prime}\right)_{t}}\right|=\prod_{r=1}^{n}\left[\frac{r !(a)_{r}}{(c)_{r}\left(c^{\prime}\right)_{r}}\right], \\
\left|\frac{(a)_{s}\left(a^{\prime}\right)_{t}}{(c)_{s+l}}\right|=(-1)^{+n(n+1)} \prod_{r=1}^{n}\left[\frac{r !(a)_{r}\left(a^{\prime}\right)_{r}}{(c+r-1)_{r}(c)_{2 r}}\right] .
\end{gathered}
$$

Of these I have given (14) elsewhere ${ }^{4}$ : (15) and (16) are more simply written as

$$
\left|(a)_{o+1}\right|=\prod_{r=1}^{n}\left[r !(a)_{r}\right]
$$

1 (7), 23 (25).

(1) (9).

(6), $185(71)$.

(1) (11). 
which is elementary, and the more recondite

$$
\left|\frac{1}{(c)_{s+l}}\right|=(-1)^{\frac{1}{2} n(n+1)} \prod_{r \rightarrow 1}^{n}\left[\frac{r !}{(c+r-1)_{r}(c)_{2 r}}\right] .
$$

\section{REFERENCES.}

1. J. L. Burchnall, "Some determinants with hypergeometric elements", Quart. J. of Math. (Oxford) (2), 3(1952), 151-157.

2. _ " An algebraic property of the classical polynomials", Proc. London Math. Soc. (3), I (1951), 232-240.

3. J. Geronimus, "On some persymmetric determinants formed by the polynomials of M. Appell ", Journal London Math. Soc., 6 (1931), 55-59.

4. J. L. Burchnall, "A note on the polynomials of Hermite", Quart. J. of Math. (Oxford), 12 (1941), 9-11.

5. J. L. Burchnall and T. W. Chaundy, "Expansions of Appell's double hypergeometric functions", Quart. J. of Math. (Oxford), 11 (1940), 249-270.

6. , "Expansions of Appell's double hypergeometric functions, II ", Quart. J. of Math. (Oxford), 12 (1941), 112-128.

7. P. Appell and J. Kampé de Fériet, Fonctions hypergéométriques et hypersphériques (Paris, 1926).

\section{The UNIVERSTTY, DURham.}

